Predicting image blur in proton radiography: comparisons between measurements and Monte Carlo simulations

A. E. Schach von Wittenau, M. B. Aufderheide, G. L. Henderson

May 12,2010

Symposium on Radiation Measurements and Applications Ann Arbor, MI, United States May 24, 2010 through May 27, 2010 
This document was prepared as an account of work sponsored by an agency of the United States government. Neither the United States government nor Lawrence Livermore National Security, LLC, nor any of their employees makes any warranty, expressed or implied, or assumes any legal liability or responsibility for the accuracy, completeness, or usefulness of any information, apparatus, product, or process disclosed, or represents that its use would not infringe privately owned rights. Reference herein to any specific commercial product, process, or service by trade name, trademark, manufacturer, or otherwise does not necessarily constitute or imply its endorsement, recommendation, or favoring by the United States government or Lawrence Livermore National Security, LLC. The views and opinions of authors expressed herein do not necessarily state or reflect those of the United States government or Lawrence Livermore National Security, LLC, and shall not be used for advertising or product endorsement purposes. 


\title{
Predicting image blur in proton radiography: comparisons between measurements and Monte Carlo simulations
}

Alexis Schach von Wittenau, Maurice Aufderheide, III, Gary Henderson

Lawrence Livermore National Laboratory, Livermore, CA 94551

\begin{abstract}
Given the cost and lead-times involved in high-energy proton radiography, it is prudent to model proposed radiographic experiments to see if the images predicted would return useful information. We recently modified our raytracing transmission radiography modeling code HADES to perform simplified Monte Carlo simulations of the transport of protons in a proton radiography beamline. Beamline objects include the initial diffuser, vacuum magnetic fields, windows, angleselecting collimators, and objects described as distorted 2D (planar or cylindrical) meshes or as distorted 3D hexahedral meshes. We present an overview of the algorithms used for the modeling and code timings for simulations through typical 2D and 3D meshes. We next calculate expected changes in image blur as scattering materials are placed upstream and downstream of a resolution test object (a $3 \mathrm{~mm}$ thick sheet of tantalum, into which $0.4 \mathrm{~mm}$ wide slits have been cut), and as the current supplied to the focusing magnets is varied. We compare and contrast the resulting simulations with the results of measurements obtained at the $800 \mathrm{MeV}$ Los Alamos LANSCE Line-C proton radiography facility.
\end{abstract}

\section{Introduction}

The radiographic imaging beamlines at proton accelerator facilities may be used to obtain multiple (up to several dozen) images of a time-varying experiment [1-8]. Figure 1 shows a schematic of a proton radiography experiment. A pulse of protons, extracted from either an accelerator or a storage ring, strikes a thin foil (the diffuser). A series of magnets next shapes the now diverging beam so that the protons illuminate an area $\sim 10$ centimeters wide at the object plane. The experiment to be radiographed is placed at this object plane. The protons traverse the user's experiment, lose a few percent of their energy, and are scattered by a few milliradians. Another series of magnets focuses the protons onto a scintillator screen placed at the image plane. Up to several dozen images may be taken, depending on the pulse structure of the protons extracted from the accelerator and on the number and types of high-speed cameras used.

Given the lead times and logistical issues involved with these experiments, the experimenter would like to be able to estimate the quality of the images expected and to identify potential pitfalls in the experimental design. We recently modified our formerly raytracing-only radiographic simulation code HADES $[9,10]$ to allow our users to perform fast, albeit reduced-physics, Monte Carlo simulations of planned proton radiographic experiments. We also obtained images of a resolution test object, taken under a variety of experimental geometries and over a range of imaging magnet settings, to benchmark our code.

\section{Sources of image degradation and effects on ex- perimental design}

Some of the image degradation is due to the proton radiography beamline itself, such as the proton scattering from and energy loss straggling in the windows and blast shields upstream or downstream of the experiment. Other sources are due to the experiment itself. For example, the object can move off the object plane. Also, the object's line-of-sight mass may change as the object expands or as pieces move with respect to each other. These processes will affect the sharpness of the image at the downstream imaging location, given that (1) the magnets are set to a user-specified strength at the beginning of the experiment and (2) the magnet strength cannot be varied during the time span of the experiment (usually several tens of microseconds).

Thus, while the user can acquire images over a range of times, the evolving nature of the experiment and the fixed nature of the magnets means that only one of the images will likely be acquired at an optimal focus, the other images may be slightly or even significantly blurred.

\section{Software implementation}

References $[6,11-15]$ discuss various aspects of proton radiography, as well as their implementation in the full-physics Monte Carlo code MCNP. We made a few additional assumptions when adding proton Monte Carlo algorithms to HADES. First, we note that the beamline collimators and imaging magnets downstream of the object are arranged to eliminate protons that have been scattered 
by more than a few milliradians $[6,15,16]$ (or which have lost a significant amount of energy). We therefore omit the tracking of inelastically-scattered protons, along with the tracking of any secondary particles that would have been produced; we instead terminate the particle track. We next note that the angles in elastic (diffractive) scattering (see, for example, [17-20]) are such that, at $800 \mathrm{MeV}$, that the downstream collimator would remove the so-affected protons. (We also note that this assumption is invalid at higher energies, see in particular [11] and in general the data and references tabulated at [18].)

Collisions between protons and collimators, and between protons and lens walls, are considered to be particleterminating events.

Our implementations of the remaining physics processes are based on discussions and algorithms published by others (e.g., Molière scattering [21, 22], energy loss [23], energy straggling in the Landau [24] and Vavilov [25] approximations). Energy-dependent cross-sections for large-angle elastic and inelastic scattering are parametrized along the lines given in $[26,27]$. Our values for the $f(A)$ described in $[26,27]$ were chosen to match the $1 \mathrm{GeV}$ parametrization given in [28].

We treat the proton radiography beamline as a sequence of adjacent, but non-overlapping objects (e.g., sheets of material for the diffuser, for windows, etc., vacuum regions within the magnet bores, and the hydrodynamic meshes used to model objects which evolve over the course of a few microseconds). ${ }^{1}$ For simulation purposes an 800 $\mathrm{MeV}$ proton is assumed incident on the upstream diffuser. The proton scatters in the diffuser and is tracked through the windows and lenses upstream of the object plane. The proton is tracked through the mesh and any downstream windows until it reaches the first downstream magnet. At this point the proton's position, direction, and energy are kept in memory as starting conditions for a mini-series of Monte Carlo runs over a user-specified range of imaging magnet settings. The proton is tracked to the image plane for each of these magnet settings. ${ }^{2}$ HADES then starts with the next proton, assumed incident on the diffuser. At the end of the simulation, then, the user has a series of images, one per magnet setting, and can choose the magnet setting which gives the most useful image.

Particle tracking through 2-dimensional cylindrical and planar meshes is performed as discussed in [31]; tracking in 3-dimensional hexahedral meshes is based on [32]. Steps larger than $600 \mu \mathrm{m}$ (up to a maximum of $1 \mathrm{~cm}$ ) are performed using the 'random-hinge' algorithm [33].

Protons are moved through vacuum regions of quadrupole magnets in the radiography beamline (see $[13,16]$ ) via COSY [34] magnet maps (order 5, fringe-field model 0 ).

The code runs on multi-CPU nodes of multinode paral-

\footnotetext{
${ }^{1}$ A stack of non-overlapping objects is also used by the Monte Carlo code BEAM [29] for modeling radiotherapy accelerators.

2 "Source particle reuse" is also used by the radiotherapy Monte Carlo code PEREGRINE [30].
}

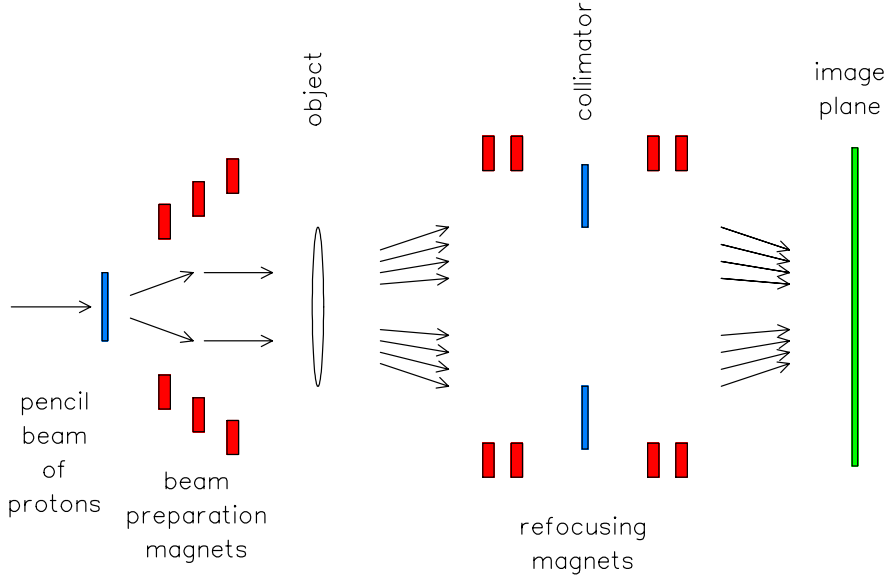

Figure 1: Stylized drawing of a proton radiography beamline.

lel computers; parallelization is performed using MPI between nodes and with threads on the individual nodes.

\section{Experiment}

Our resolution test object (RTO) is a $3 \mathrm{~mm}$ thick sheet of tantalum, into which slits of $0.4 \mathrm{~mm}$ nominal width were cut. The RTO was placed at the object location of the Line-C proton radiography beamline $(M=-1$ lens, 10 milliradian collimator), and images were acquired as a function of the current supplied to the downstream imaging magnets. Next, blocks of different thicknesses $(4 \mathrm{~cm}$, $8 \mathrm{~cm}$ ) of aluminum and polyethylene $(7 \mathrm{~cm})$ were placed upstream and downstream of the RTO, and again images were acquired as the current supplied to the imaging magnets was swept. The $330+$ images were acquired over several days; additionally on each day dark-field (i.e., no beam) and bright-field (i.e., no object) images were acquired to characterize the fixed-pattern background and the efficiency, respectively, of the cameras. The number of protons used for any given image varied between $1.3 \times 10^{9}$ and $2.1 \times 10^{9}$ protons. The relative proton count for a given image is estimated to be accurate to better than $1.5 \%$.

The raw images were converted to transmission images by first subtracting the fixed pattern background and next dividing by the beam profile. Lineouts from the central $\pm 9 \mathrm{~mm}$ portions of the images were averaged and used for further study.

\section{Simulations}

HADES was used to simulated the various combinations of RTO and upstream or downstream blocks mentioned in Section 4. Approximately $2 \times 10^{9}$ protons were used for each simulation, with 80 images being calculated over the range of magnet currents used for the particular experimental geometry. The calculated images were flattened, lineouts from the central $\pm 1 \mathrm{~cm}$ were averaged, the results were blurred to take into account the finite resolution of the imaging cameras and used for further study. 


\section{Discussion}

Fig. 2 shows lineouts from three of the experimental and calculated images of the RTO for the magnet currents shown. The measured widths are well-reproduced by the calculations for each of the settings, as are the different shapes of the dimples on each side of the main lines. Quantitatively, the HADES simulations show a slightly lower transmission than measured $(\sim 31 \%$ versus $\sim 34 \%$, respectively, in the regions between the slits in the RTO).

Fig. 3 shows lineouts from three of the experimental and calculated images when a $4-\mathrm{cm}$ thick block of aluminum is placed just downstream of the RTO for the magnet currents shown. As expected, compared with Fig. 2, the overall transmission is lower. The HADES simulations again track the changes in the shapes and heights of the lines as the magnet current is varied. As with Fig. 2, HADES predicts a transmission that is slightly lower than measured (here, $\sim 19.5 \%$ versus $\sim 21.7 \%$, respectively).

The data sets not shown here (i.e., with the aluminum blocks upstream of the RTO, as well as the sets taken with the polyethylene block) show similar behavior; that is, the observed line widths and heights are well matched while the measured transmissions are slightly higher than the calculated transmissions. The discrepancy in transmission is a systematic relative error on the order of $7 \%$. Possible sources of this disagreement (e.g., errors in data reduction, cross sections, beamline geometry model, neglect of scattered particles, assumption of perfect attenuation by the downstream collimator, treatment of the imaging lenses, etc.) are currently being investigated.

The purpose of HADES is to warn the user of changes in image quality as the experimental object evolves, and to indicate appropriate changes in magnet settings to recover, to the extent possible, the image sharpness. Figure 4 shows the HADES predictions of (1) the changes in transmission and in image quality as scattering material is put into the beamline, in this case, an 8-cm block of aluminum, just downstream of the RTO. Adding the 8 -cm aluminum block slightly more than halves the transmission. The added material also changes the energy lost by the protons. If the magnet current is left unchanged, the imaging system would give a highly-blurred image as shown. The magnets can be retuned to give a sharper image, albeit still at the overall $\sim 15 \%$ transmission.

\section{Conclusions}

We have added reduced-physics Monte Carlo tracking of protons to our radiographic simulation code HADES. Comparison of HADES simulations with a series of staticobject measurements shows that the algorithms and approximations give good agreement in terms of image sharpness over a wide range of experimental geometries and imaging-magnet settings. The calculated transmissions of the various images are slightly lower than the observed transmissions.

\section{Acknowledgments}

We have benefited enormously from the numerous discussions about proton radiography we have had with colleagues at Los Alamos National Laboratory. In particular we would like to thank John Zumbro, Chris Morris, Nick King, Fesseha Mariam, Andy Saunders and Frank Merrill.

We thank John Zumbro and Fesseha Mariam for discussions on the use of COSY as well as for their COSY input decks for various Line-C lens configurations.

We would like to thank the Line-C experimental group (B. Brooks, E. Campos, J. Dominguez, C. Espinoza, J. Fait, G. Hogan, B. Hollander, N. King, K. Kwiatkowski, D. Lewis, J. Lopez, F. Mariam, A. Meidinger, F. Merrill, D. Morley, C. Morris, M. Murray, P. Nedrow, A. Saunders, C. Schwartz, T. N. Thompson, D. Tupa, J. Tybo) for the acquisition of the data described in this paper.

This work performed under the auspices of the U.S. Department of Energy by Lawrence Livermore National Laboratory under Contract DE-AC52-07NA27344.

\section{References}

[1] P. W. Lisowski and K. F. Schoenberg Nucl. Inst. Meth. A562 (2006) 910.

[2] N. S. P. King et al. Nucl. Inst. Meth. A424 (1999) 84.

[3] A. A. Golubev et al. Atomic Energy 104 (2008) 134.

[4] L. Smilowitz et al. Phys. Rev. Lett. 100 (2008) 228301.

[5] P. A. Rigg et al. Phys. Rev. B 77 (2008) 220101.

[6] H. J. Ziock et al. Tech. Rep. LA-UR-98-1368 Los Alamos National Laboratory (1998).

[7] S. A. Koleshnikov et al. High Press. Res. 30 (2010) 83.

[8] A. A. Golubev et al. Tech. Phys. Lett. 36 (2010) 177.

[9] A. E. Schach von Wittenau et al. Med. Phys. 29 (2002) 2559.

[10] M. B. Aufderheide, III et al. in Rev. Prog. Quant. Nondest. Eval., Vol. 20A, (2001) 507.

[11] J. D. Zumbro Nucl. Inst. Meth. B246 (2006) 479.

[12] J. D. Zumbro et al. Radia. Prot. Dosim. 117 (2005) 447.

[13] J. S. Bull et al. Radia. Prot. Dosim. 116 (2005) 307.

[14] H. G. Hughes et al. Radia. Prot. Dosim. 116 (2005) 109.

[15] C. T. Mottershead and J. D. Zumbro in Proc. 1997 Part. Accel. Conf. (IEEE, 1998) 1397.

[16] J. A. Favorite et al. Trans. Am. Nucl. Soc. 83 (2000) 338.

[17] R. Bertini et al. Phys. Lett. 45B (1973) 119.

[18] http://www.nea.fr/dbdata/x4/.

[19] G. S. Blanpied et al. Phys. Rev. C 18 (1973) 1436.

[20] G. D. Alkhazov et al. JETP Lett. 18 (1973) 181.

[21] P. Andreo et al. Med. Phys. 20 (1993) 1315.

[22] B. Gottschalk et al. Nucl. Instr. Meth. B74 (1993) 467.

[23] J. F. Ziegler Nucl. Inst. Meth. B219-220 (2004) 1027.

[24] H. G. Hughes Tech. Rep. LA-UR-96-4583 Los Alamos National Laboratory (1996).

[25] O. Chibani IEEE Trans. Nucl. Sci. 45 (1998) 2288.

[26] V. Grichine Nucl. Inst. Meth. B267 (2009) 2460.

[27] V. Grichine Eur. Phys. J. C62 (2009) 399.

[28] G. Igo et al. Nucl. Phys. B3 (1967) 181.

[29] D. W. O. Rogers et al. Med. Phys. 22 (1995) 503

[30] C. L. Hartmann Siantar et al. Med. Phys. 28 (2001) 1322.

[31] A. F. Bielajew Tech. Rep. PIRS-0341 Nat. Res. Council Canada (1996).

[32] G. Marmitt and P. Slusallek in EuroVis06 (Eurographics Assoc., 2006) 235

[33] J. M. Fernández-Varea et al. Nucl. Instr. Meth. B73 (1993) 447.

[34] K. Makino and M. Berz Nucl. Inst. Meth. A558 (2006) 346. 

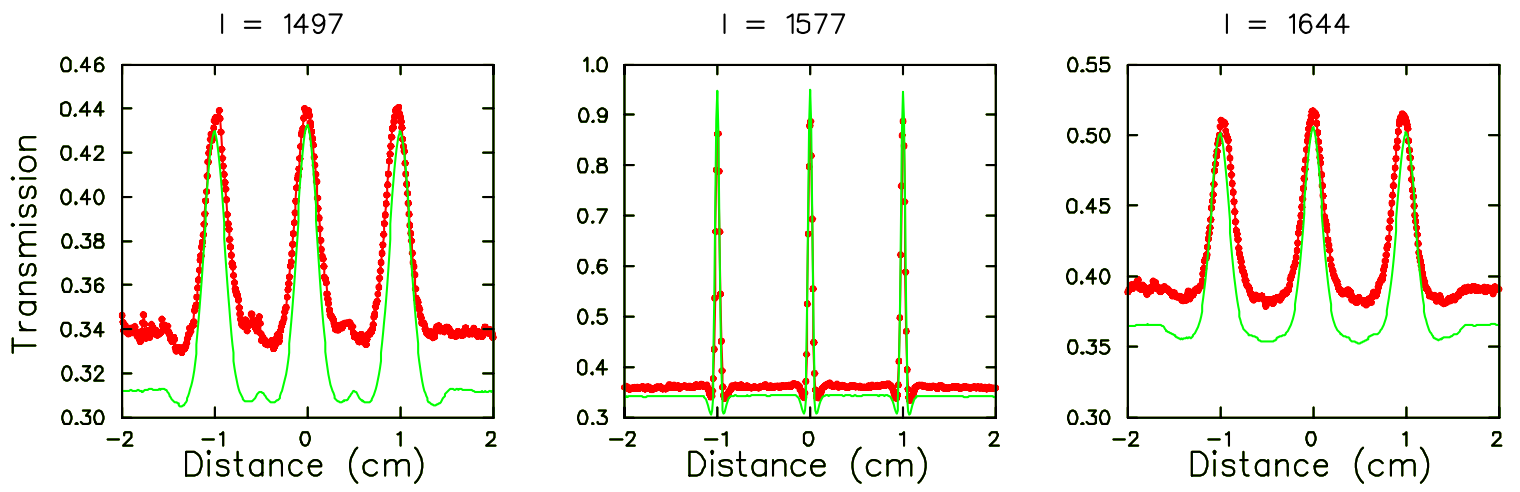

Figure 2: Lineouts through proton radiographs of the resolution test object for the given imaging magnet currents. Red dotted curves: experiment. Green solid curves: HADES simulations. Note that the vertical scale changes from panel to panel. (In this and subsequent figures, the imaging magnet currents are given in amperes; the magnets upstream of the objects were left unchanged.)
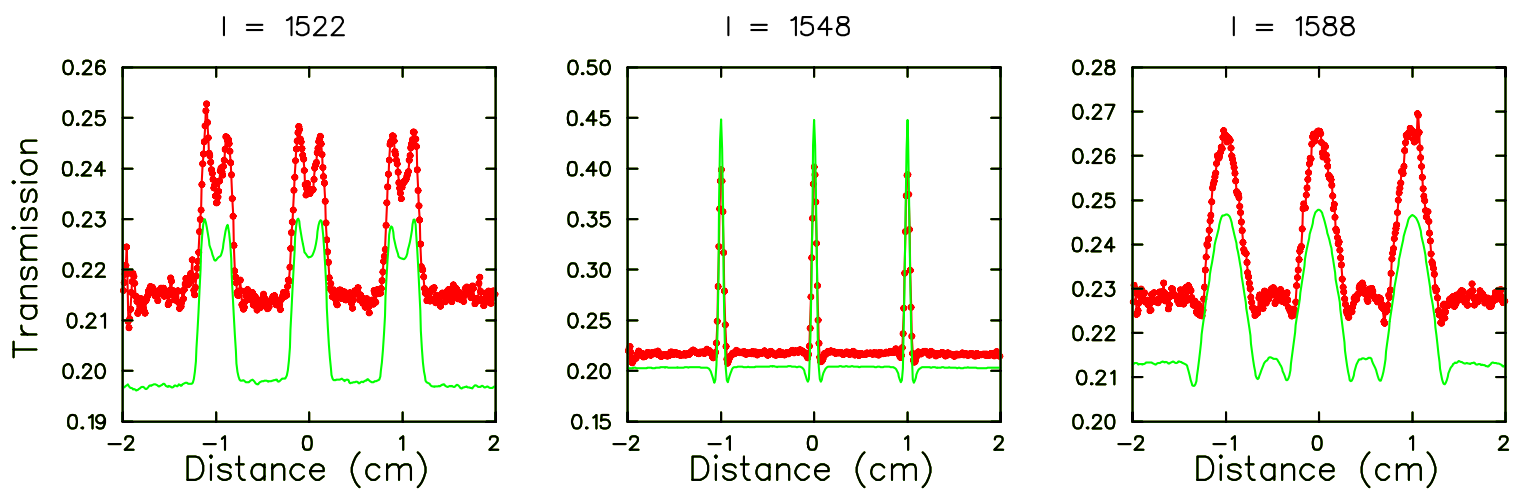

Figure 3: Lineouts through proton radiographs for the given magnet currents of the resolution test object with a downstream 4-cm thick block of aluminum. Red dotted curves: experiment. Green solid curves: HADES simulations. Note that the vertical scale changes from panel to panel.
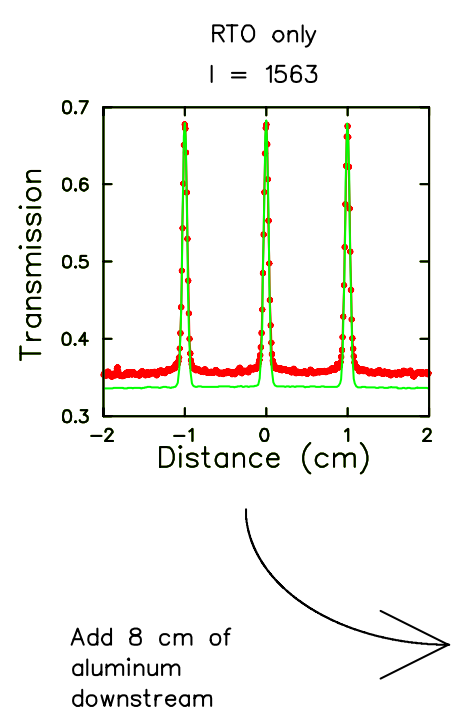
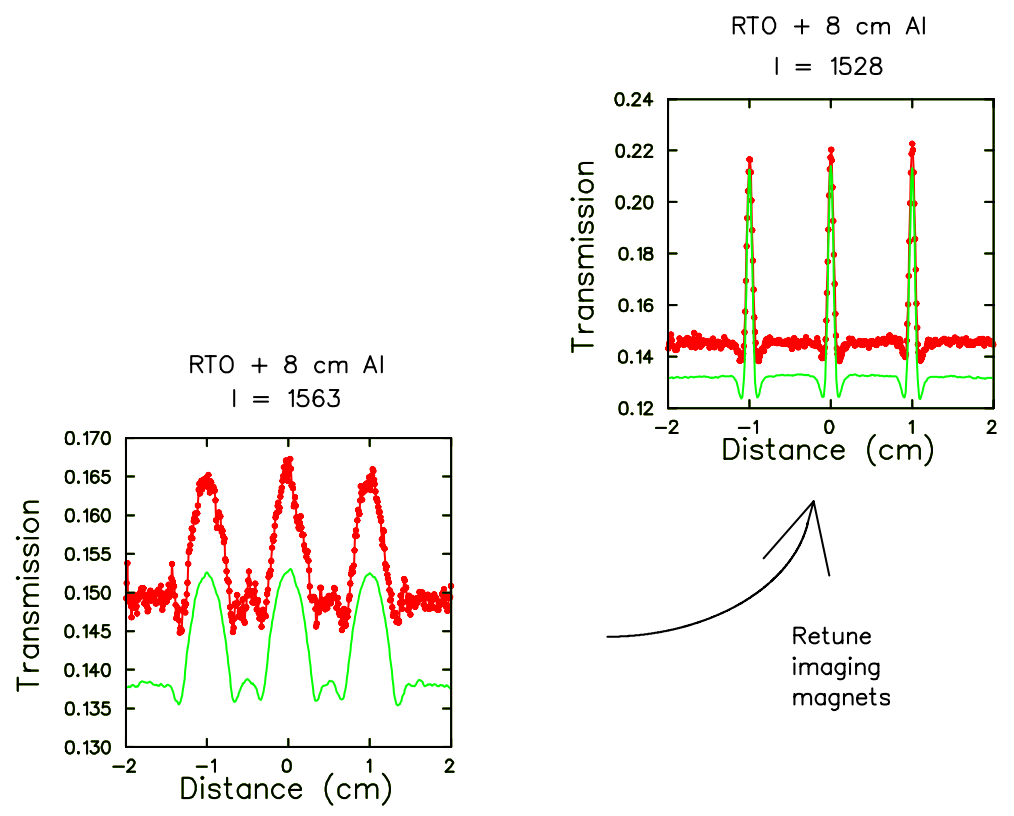

Figure 4: Adding downstream material blurs the image. Reducing the current in the focusing magnets recovers much of the image quality. Red dotted curves: experiment. Green solid curves: HADES simulations. Note that the vertical scale changes from panel to panel. 